(C) 2016 IEEE. Personal use of this material is permitted. Permission from IEEE must be obtained for all other uses, in any current or future media, including reprinting/republishing this material for advertising or promotional purposes, creating new collective works, for resale or redistribution to servers or lists, or reuse of any copyrighted component of this work in other works. 


\title{
Performance Comparison of Packet Scheduling Algorithms in LTE-A HetNets
}

\author{
Ramprasad Subramanian", Dr. Kumbesan Sandrasegaran ${ }^{*}$, Dr. Xiaoying Kong* \\ *Centre for Real Time Information Networks \\ School of Computing and Communication \\ Faculty of Engineering and Information Technology, University of Technology Sydney \\ Sydney, Australia
}

\begin{abstract}
Performance comparison of various Packet Scheduling (PS) algorithms such as Proportional Fair (PF), Maximum Largest Weighted Delay First (MLWDF) and Exponential/Proportional Fair (EXP/PF) has been studied in HetNets environment. The performance indicators such as throughput, Packet Loss Ratio (PLR), delay and fairness are considered to judge the performance of the scheduling algorithms. Various strategies such as increasing the number of pico cells in the cell edge were used in the simulation for the performance evaluation study. The results achieved by various simulations show that adding the pico cells to the existing macros enhances the overall system performance in addition to various scheduling algorithms implemented in macros. Simulation results show that the overall system gain has increased by adding picos, provide better coverage in the cell edge and increase the capacity of the network to provide better Quality of Service (QoS). Furthermore, simulations show that MLWDF performs better for video traffic than compared to other with PS algorithms.
\end{abstract}

Keywords-Packer scheduling; packet loss ratio; delay; fairness; pico; femto; macro; QoS; cell edge; downlink; Lte-Sim;

\section{INTRODUCTION}

The 3GPP has proposed the LTE system to satisfy the requirements to provide high capacity, large coverage area, high bit rates and lower delay etc. Different methods have been adopted in $4 \mathrm{G}$ mobile networks to improve the LTE system and meet the aforementioned requirement. The most cost-effective solution to achieve these demands is by introducing HetNets. HetNets can be defined as the mixture of maintaining micro (such as pico and femto cells) cells working alongside the macro network [1]. The implementation of HetNets is classified as macro with pico and macro with femto [1]. In the HetNets, the same carrier frequency is utilized in both macro and its associated small cells (pico or femto) to efficiently utilize the available resources and thereby saving the operational cost. In LTE/LTE-A, the eNB is in responsible of all functionalities of Radio Resource Management (RRM) such as Admission Control (AC), Power Control (PC), Handover Control (HOC), Congestion Control (CC) or Load Control (LC), interference coordination and control (ICC) and Packet Scheduling (PS). In this paper, the PS algorithms are dealt in detail. PS provides can provide QoS guarantee because of the efficient and the best usage of available radio resources. Additionally, the responsibility of transmitting the user's data packets in the telecommunication system is manipulated by PS algorithms [2]. Scheduling algorithms are a vendor specific with no standardisations specified by $3 \mathrm{GPP}$ and therefore they are based on the network capability and user's demands. This has led to the proposal of different PS algorithms according to the type of traffic, QoS requirement, vendor capability and applied services. Some of the most wellknown PS schemes such as PF, MLWDF and EXP/PF algorithms [3][4][5] are studied in this paper.

The rest of this paper is organized as follows. Section 2 gives an overview of downlink (DL) system model of LTE. In Section 3 detailed description of the packet scheduling algorithms such as PF, MLWDF and EXP/PF have been provided. The simulation environment is described in Section 4. In Section 5, the results obtained from the simulations are discussed and conclusion is provided in Section 6.

The uniqueness of the paper is that to the best of our knowledge, the developers of this simulator have not developed simulation scenarios focusing on macro and pico cells. Hence, an attempt has been made to develop these scenarios and performance evaluations of popular PS algorithms have been performed.

\section{LTE SYSTEM MODEL IN DL DIRECTION}

In the downlink direction of the LTE system, the basic element of radio resource is called Resource Block (RB). The User Equipment (UE) is allocated with certain number of available RBs based on the user channel condition, required services, and the agreed QoS requirements. RB could be defined in both frequency domain and time domain. In frequency domain, $\mathrm{RB}$ is allocated with 12 of $15 \mathrm{kHz}$ contiguous subcarriers consisting of $180 \mathrm{kHz}$ as total bandwidth for one RB. On the other hand, the time domain RB is allocated with 7 symbols of OFDMA (Orthogonal Frequency Division Multiple Access) in a single $0.5 \mathrm{~ms}$ time slot [7].

As aforementioned before, eNB performs PS besides other RRM operations in LTE system. On the other hand, the bandwidth (BW) that can be used in the LTE system is in the rage of $5-20 \mathrm{MHz}$. In this study, the $\mathrm{BW}$ utilization is 10 $\mathrm{MHz}$. Since the same carrier frequency is used in macro and its associated micro (pico or femto), LTE suffers from InterCell Interference (ICI). Packet scheduling operation is performed by eNB for every $1 \mathrm{~ms}$ Transmission Time Interval (TTI). Hence, two of $0.5 \mathrm{~ms}(1 \mathrm{~ms})$ is the time that the UE have to wait before it is allocated with new RBs. Each time the $\mathrm{UE}$ is assigned with two RBs because each RB is $0.5 \mathrm{~ms}$ in the time domain. The eNB takes the scheduling decision at each 
TTI after considering the feedback Channel Quality Indicator (CQI) reports from UE in uplink (UL). The CQI mainly comprise of the channel condition, and more specifically, the Signal to Noise Ratio (SNR) and the type of service requested. The SNR value is beneficial for the serving eNB to decide the downlink bit rate for each connected user in each $1 \mathrm{~ms}$ scheduling time. As an example, the number of bits involved in 2 contiguous RBs is calculated in [7].

Equation 1 [8] determines the data rate $d r_{i}(t)$ for the user $i$ at $j$ sub-carrier on the $\mathrm{RB}$ and at $t$ time. The data rate as specified in equation 1 is determined by the serving eNB on basis of the SNR values reported by the UE in the feedback reports.

$$
\begin{aligned}
& d r_{i}(t)=n b s * n s s * n s t * n s r \\
& \mathrm{nbs}=n^{2} \text { bits }_{i, j}(t) / \text { symbol } \\
& \mathrm{nss}=n s y m b o l s / \text { slot } \\
& \mathrm{nts}=n s l o t s / T T I \\
& \mathrm{nsr}=n s c / R B \mathrm{rgg}
\end{aligned}
$$

$n b s$ is the number of bits per symbol. nss is the number of symbols per slot. While $n t s$ represents the number of slots per TTI, nsr clarifies how many sub-carriers per RB. Table 1 summarizes the mapping between SNR values and their associated data rates values.

TABLE I. MAPPING BETWEEN INSTANTANEOUS DOWNLINK SNR AND DATA RATES

\begin{tabular}{|c|c|c|}
\hline $\begin{array}{c}\text { Minimum SNR } \\
\text { level }(\mathrm{dB})\end{array}$ & $\begin{array}{c}\text { Modulation and } \\
\text { coding }\end{array}$ & $\begin{array}{c}\text { Data rate } \\
(\mathrm{Kbps})\end{array}$ \\
\hline 1.7 & QPSK $(1 / 2)$ & 168 \\
\hline 3.7 & QPSK(2/3) & 224 \\
\hline 4.5 & QPSK(3/4) & 252 \\
\hline 7.2 & 16 QAM (1/2) & 336 \\
\hline 9.5 & 16 QAM (2/3) & 448 \\
\hline 10.7 & 16 QAM (3/4) & 504 \\
\hline 14.8 & 64 QAM (2/3) & 672 \\
\hline 16.1 & 64 QAM (3/4) & 756 \\
\hline
\end{tabular}

As the user's packets arrive, the serving eNB buffers them in a particular buffer for each active user. In the buffer, the eNB assigns a timestamp for each queued packet in order to process them by the scheduler or drop them, in case the maximum waiting time of the packet in the queue is reached. After that, First-In-First-Out (FIFO) technique is applied by the eNB to transmit the scheduled packets to their associated UEs in the DL direction.

PS is an element of eNB that is responsible for the scheduling operation. Additionally, at each TTI, the scheduler prioritizes and classifies the packets sent by the active users based on the applied scheduling algorithm. Various algorithms specify various criteria's that are used to make the scheduling decision. These criteria's involve buffer status, channel condition, Head-Of-Line (HOL) packet delay, and service type. Based on the channel status, RB are assigned for UEs and high priority UEs are selected for scheduling and allocated one or more RBs based on the requested service and status conditions.

\section{PACKET SCHEDULING ALGORITHMS}

The PS algorithms are designed to utilize available radio resources efficiently, to ensure fairness among scheduled UEs and to satisfy the QoS requirements [10]. The PS schemes that are used in this paper are designed to support both Real Time (RT) and Non-Real Time (NRT) traffic.

\section{A. PF Algorithm}

The PF is proposed to support RT traffic with fairness [3]. It is applied in Code Division Multiple Access- High Data Rate (CDMA-HDR) system. There is a trade-off between fairness among users and the total system throughput in this algorithm. In order to allocate RBs, this scheme considers the past data rate and the conditions of the channel. PF algorithm assigns radio resource blocks for the scheduled user if the user maximizes the metric $k$. It is calculated as the ratio of reachable data rate $r_{i}(t)$ of user $i$ at time $t$ and average data rate of the same user at the same time interval.

$$
\begin{aligned}
& k=\arg \max \frac{r_{i}(t)}{R_{i}(t)} \\
& R_{i}(t)=\left(1-\frac{1}{t_{c}}\right) * R_{i}(t-1)+\frac{1}{t_{c}} * r_{i}(t-1)
\end{aligned}
$$

In order to maximize the fairness and throughput for any scheduled user, the window size $t_{c}$ is used to update the past data rate values. $r_{i}(t-1)=0$ unless user $i$ is selected for transmission at $(t-1)$.

\section{B. MLWDF Algorithm}

MLWDF is introduced in order to support RT data users [4]. It is used in CDMA-HDR system. The algorithm is more complex than PF. MLWDF is utilized in the scenario where different requirements of QoS are crucial. The reason behind the complexity of MLWDF is that while assigning RBs, it takes channel variations into account. Moreover, the time delay is taken into consideration for the video traffic. The RBs is granted to the users in MLWDF if it maximizes the below equation.

$$
k=\arg \max a_{i} W_{i}(t) \frac{r_{i}(t)}{R_{i}(t)}
$$

Where $a_{i}=-\frac{\left(\log \delta_{i}\right)}{\tau_{i}}$

$W_{i}(t)$, denotes the difference in time between current and arrival times of the packet that is known as the head of line (HOL) packet delay of the user $i$ at time $t$. The average data rate of the user at the time interval $t$ is $R_{i}(t)$, while the achievable data rate of the user $i$ at time $t$ is $r_{i}(t) . \tau_{i}$ is the delay threshold for a packet of user $i$ and $\delta_{i}$ is the maximum HOL packet delay probability of user $i$. The latter is considered in order to exceed the delay threshold of user $i$.

\section{EXP/PF Algorithm}

$\mathrm{EXP} / \mathrm{PF}$ is an enhancement of the PF scheduling algorithm [6]. This algorithm is mainly designed to support multimedia applications. It was proposed in the Adaptive Modulation and 
Coding and Time Division Multiplexing (AMC/TDM) systems. In fact, EXP/PF algorithm is not only designed for different sorts of RT services, but also for NRT services. The metric $k$ is introduced for both RT and Non-RT in which RBs is assigned to the users based on $k$.

$k=\arg \max \begin{cases}\exp \left(\frac{a_{i} W_{i}(t)-a \overline{W(t)}}{1+\sqrt{a W(t)}}\right) \frac{r_{i}(t)}{R_{i}(t)} & i \in R T \\ \frac{w(t)}{M(t)} \frac{r_{i}(t)}{R_{i}(t)} & i \in N R T\end{cases}$

Where,

$a \overline{w(t)}=\frac{1}{N_{R T}} \sum_{i \in R T} a_{i} W_{i}(t)$

$w(t)=\left\{\begin{array}{c}w(t-1)-\varepsilon \quad W_{\max }>\tau_{\max } \\ w(t-1)+\frac{\varepsilon}{k} W_{\max }<\tau_{\max }\end{array}\right.$

The average number of packets in the eNB buffer at time $t$ is denoted by $M(t)$. In equation 8 , the symbols $k$ and $\varepsilon$ are constants. $W_{i}(t)$ is explained in MLWDF, $W_{\max }$ is the HOL packets delay of RT service and $\tau_{\max }$ is the maximum delay for RT service users. The EXP/PF gives priority to the RT traffic users than NRT traffic users if the HOL values are reaching the delay threshold.

\section{SIMULATION ENVIORNMENT}

The entire analysis and study in this paper was performed by using LTE-Sim version 5. To the best of our knowledge LTESim has not yet developed any code for HetNets environment comprising of macro and pico cells. The code developed in this paper could be considered as an enhancement in the upcoming release of the LTE-Sim. However, LTE-Sim has developed substantial scenario that can be used to simulate and examine macro with femto HetNets environment. Our paper is based on the scenario where a single Macro cell is surrounded by 2,4,6,8 and 10 small, reduced power Pico cells in the cell edge. As the number of picos is added to the system at the cell edge, it is expected to enhance the system performance. However, by increasing the number of pico cells without proper planning, it will result in the increase of ICI, since the same carrier frequency is used by both macro and pico cells. The increase in ICI will confine the gain obtained by adding more pico cells in the cell edge [2]. The simulation environment is shown Figure.3, where it shows the distribution of pico cells in the edges of the macro cell environment. In our simulations we have considered a macro cell with the radius of $1 \mathrm{~km}$ and all the pico cells with $0.1 \mathrm{~km}$ located on the edge of the macro cell. The latitude and longitude of the pico cells are not presented in this paper due to space constrains but in original simulation these parameters were considered. Table II shows the simulation parameters that are considered in simulation. This ensures that all new results of the system performance come from the factor of adding 2 Pico cells only. Users at pico cells are not equal to the macro cell. Pico cannot serve more than 30 users at a time [10], thereby, after 30 users there are no more users could be added to any pico cells in the simulation. Nevertheless, all cells start with 10 users and are increased by the factor of 2 . The data flow is equally divided between video and voice applications with each application constituting $50 \%$ of the total system traffics.

This design is chosen because it has lot of analogies to the real time system aimed to cover larger area and more users, especially the users in the cell edge where they suffer from lack of connectivity with macro cell. The ICI is modeled, handover is activated, non-uniform user distribution within the cells is applied, and $3 \mathrm{~km} / \mathrm{h}$ constant speed is considered as the user speed. In addition, the 3GPP urban macro cell propagation loss model has been implemented including pathloss, penetration loss, interference, multi-path loss and shadow fading which are summarized below:

- Path loss: $128.1+3.76 \log _{10}(d), d$ refers the distance between the eNB and the user in kilometers.

- Penetration loss: $10 \mathrm{~dB}$

- Multipath loss: using one of the well-known methods called Jakes model

- Shadow fading loss: log-normal distribution

- Mean value of $0 \mathrm{~dB}$.

- $\quad$ Standard deviation of $10 \mathrm{~dB}$.

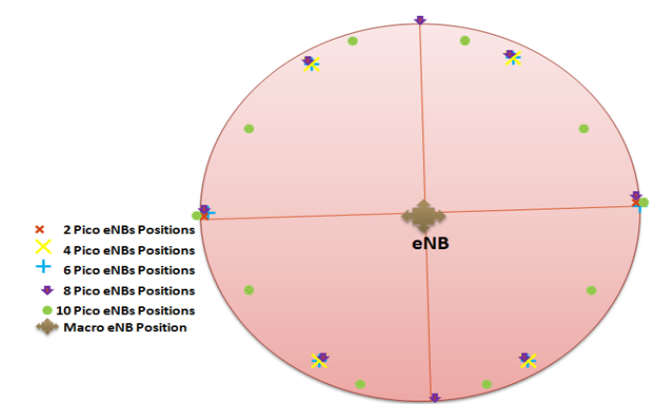

Fig.3. Applied HetNets (Macro with Multiple Picos Scenarios)

The system performance is evaluated in all aforementioned algorithms based on packet throughput as defined in equation 9, PLR as shown in equation 10, packet delay (latency) and fairness index as seen in equation 11. Jain's method is applied for fairness among users [13]. According to [17], the value of 1 is the optimum value for the best fair scenario among the scheduled users in which the resources are shared appropriately. Fairness index can be calculated as value 1 minus the value of the difference between the maximum and minimum size of transmitted packets of the most and least scheduled users. Equation (11) is used for calculating the fairness value [1] and [17].

$$
\begin{aligned}
& \text { throughput }=\frac{1}{T} \sum_{i=1}^{K} \sum_{t=1}^{T} \operatorname{ptransmit}_{i}(t) \\
& P L R=\frac{\sum_{i=1}^{K} \sum_{t=1}^{T} \operatorname{pdiscard}_{i}(t)}{\sum_{i=1}^{K} \Sigma_{t=1}^{T} \operatorname{psize}_{i}(t)} \\
& \text { fairness }=1-\frac{\text { ptotaltransmit }_{\max }-\text { ptotaltransmit }_{\min }}{\sum_{i=1}^{K} \sum_{t=1}^{T} p \operatorname{size}_{i}(t)}
\end{aligned}
$$


The $\operatorname{ptransmit}_{i}(t)$ is the size of transmitted packets, $\operatorname{pdiscard}_{i}(t)$ is the size of discarded or lost packets during the connection, psize $_{i}$ is the summation of all arrived packets that are buffered into serving eNB [9]. The aforementioned total size of transmitted packets of the best served UE and the worse served UE are represented in equation 11 as ptotaltransmit $_{\max }$ and ptotaltransmit min $_{\text {. In }}$ order to obtain more accurate results five simulations have been performed for each algorithm (PF, MLWDF and EXP) with different number of users such as 10, 20, 30, 40, 50, 60, 70 and 80 . For each case 2, 4, 6, and 8 Picos cells are considered. This yielded a total of 2040 simulation outcomes. The average values have been taken to draw the simulation graphs at each point of users for each case of number of picos.

TABLE II. SIMULATION PARAMETERS

\begin{tabular}{|c|c|}
\hline Simulation parameter & Values \\
\hline Simulation time & $40 \mathrm{~s}$ \\
\hline Flow duration & $30 \mathrm{~s}$ \\
\hline Slot duration & $0.5 \mathrm{~ms}$ \\
\hline TTI & $1 \mathrm{~ms}$ \\
\hline Number of OFDM symbols/slots & 7 \\
\hline Macro cell radium & $1 \mathrm{~km}$ \\
\hline Macro eNB power & $49 \mathrm{dBm}$ \\
\hline Pico eNB power & $30 \mathrm{dBm}$ \\
\hline User speed & $3 \mathrm{Km} / \mathrm{h}$ \\
\hline VoIP bit rate & $8.4 \mathrm{kbps}$ \\
\hline Video bit rate & $242 \mathrm{kbps}$ \\
\hline Frame structure type & $\mathrm{FDD}$ \\
\hline Bandwidth & $10 \mathrm{MHz}$ \\
\hline Number of RB & 50 \\
\hline No of subcarriers/RBs & 12 \\
\hline Subcarrier spacing & $15 \mathrm{KHz}$ \\
\hline
\end{tabular}

\section{SimUlation RESUlts}

As mentioned before, adding more picos more likely enhances the system performance. This is proven in this simulation demonstrated through the throughput, PLR, delay, and fairness. Adding two extra pico cells improves the overall system throughput with an absolute value. On the flip side, increasing the number of users increases the effect of ICI.

\section{A. Throughput}

The average overall system throughput for all scenarios is shown in Figure.4. Adding two Pico cells provides almost constant gain in all applied scheduling schemes. To explain that, PF algorithm is taken as an example. Vertically, the number of user's increases by factor of 10 such as 10, 20 and 30 and the gain value of 3.39 Mbps also doubles almost proportionally with the increase in UEs. For example, at 20 users the average value for all scenarios is $6.78 \mathrm{Mbps}$ that is almost the double the value of $3.39 \mathrm{Mbps}$ at 10 users. The difference in the average gain between 30 users to 20 users is also 3.39 Mbps. This proves that by adding 10 users the throughput increases in a constant value due to the scheduling effect. However, the pico cells will not serve beyond 30 users and hence even if the users are accepted in pico, the gain will remain the same. However, macro cell can accept more users to the system that boosts the throughput slightly with nearly 1 Mbps caused by the effect of scheduling algorithms. For instance, the overall system gain with 40 users is at 16.41 Mbps and even when the numbers of users jump to 50, the overall system gain increases to $17.26 \mathrm{Mbps}$ and thus increasing the gain by just 1 Mbps. Horizontally, moving up from 2 to 10 Pico scenarios the throughput value increases by average gain of $10 \mathrm{Mbps}$. This value comes from the effect of adding two Pico cells to the system. That is, at the point of 40 users, at 2 pico cells the total throughput of the system is $16.41 \mathrm{Mbps}$, whereas it is 26.44 at 4 Pico cells. Hence, the difference between these two cases is almost 10 Mbps. Figure. 5 gives another view of the gain and shows clearly the system performance in each case. All aforementioned values of the gain are not constant, and they are based on the simulation parameters and system environment. The PF algorithm has a lowest throughput compared to MLWDF and EXP/PF. Figure 5 shows when the number of users' increases, the throughput of PF is slightly degraded compared with the other algorithms. For example, while the numbers of users are 60, 70 and 80, the PF results of the throughput are lower than the rest of the PS schemes. However, the behaviors of all schemes are similar to all pico scenarios. It is easy to notice that the gap (gain) between scenario 2 and 4 Picos is the same as the gap between scenario 4 and 6 picos, 6 and 8 Picos, and 8 and 10 Picos.



Fig.4. Throughput gain of video traffic in macro with 2-10 Picos cells

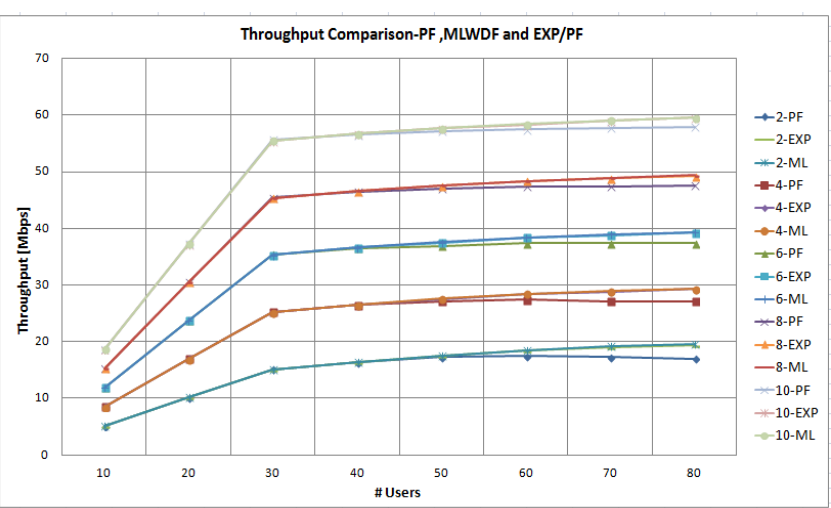

Fig.5. Throughput gain comparison of PS algorithms for video traffic in macro with 2-10 Picos cells 


\section{B. Packet Loss Ratio (PLR)}

The increase in number of users increases PLR accordingly. The PLR start to decrease by the increase in the number of pico cells. But again, increasing the pico cells will increase the number of users and thereby increases PLR. For instance, PF algorithm is the lowest performance than other algorithms in most of the cases, in which the PLR starts going up as the number of users in the system increases. The performance of PLR in the PF can be seen in Figure 6 (3D view). In this figure we can observe that, at 50 users and PF with 8 pico cells have similar PLR values like other schemes such as MLWDF and EXP/PF in two pico cells scenarios. This enforces the idea of compensation that is, adding more picos enhances system's PLR under PF. Comparing the PS schemes, the PF has the lower PLR value in all pico cell scenarios. The highest and worst PLR value of PF is in the scenario with two Pico cells as shown in Figure.6. This value is enhanced gradually when new Pico cells are added to the system. MLWDF and EXP/PF have the better PLR values in the same scenario when the number of pico cells is 10 .
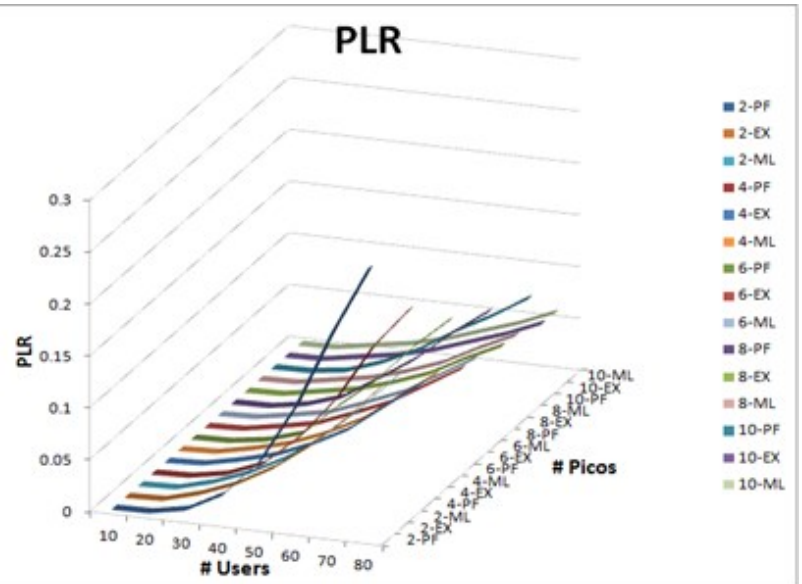

Fig.6. PLR Video traffic Comparison in Macro with 2-10 Picos Scenarios

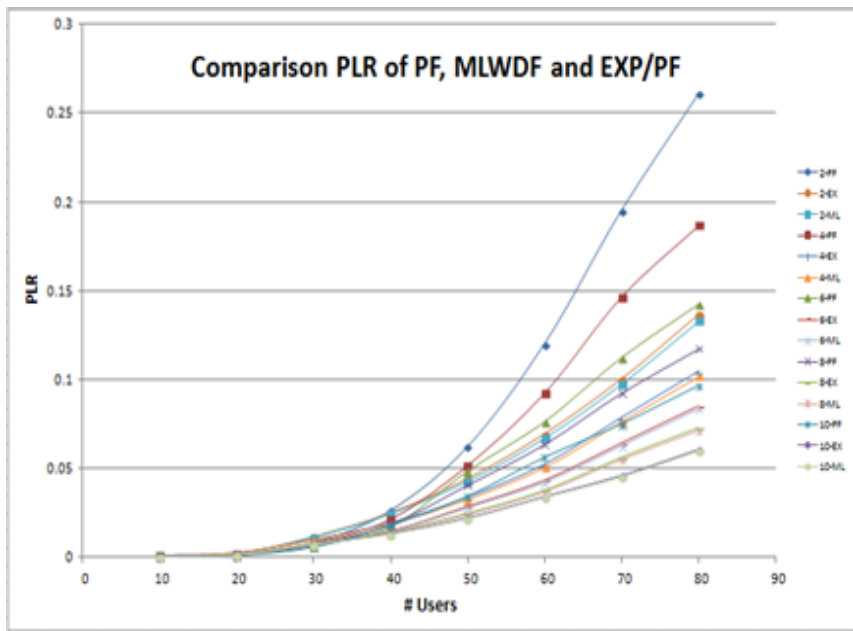

Fig.7. PLR video traffic comparison between PS algorithms

\section{PF Delay}

Delay follows similar behavior to the PLR, as the number of user's increases, the delay gets higher. Reverse of that, while pico cells are added to the system, the delay becomes lower. Figure 8 and Figure 9 shows the simulation results of PF delay. In vertical column with number of pico cells, shows high delay for PF algorithm with 2 Pico cells and the highest delay are observed when there are 80 users in two Pico cell environments. Gradually, all the PS algorithms show increasing delay as the number of user's increases. For example, at 10 users in the two Pico cells, the delay value is 6 $\mathrm{ms}$, but the delay value increases to $10.32 \mathrm{~ms}$ when the number of users increases to 20 users in the same two Pico cells. To overwhelm that, filling the system with more picos that is proven by the simulation can decrease the delay values. Horizontally, it is easy to notice that PF delay starts decreasing while the number of pico cells increases.

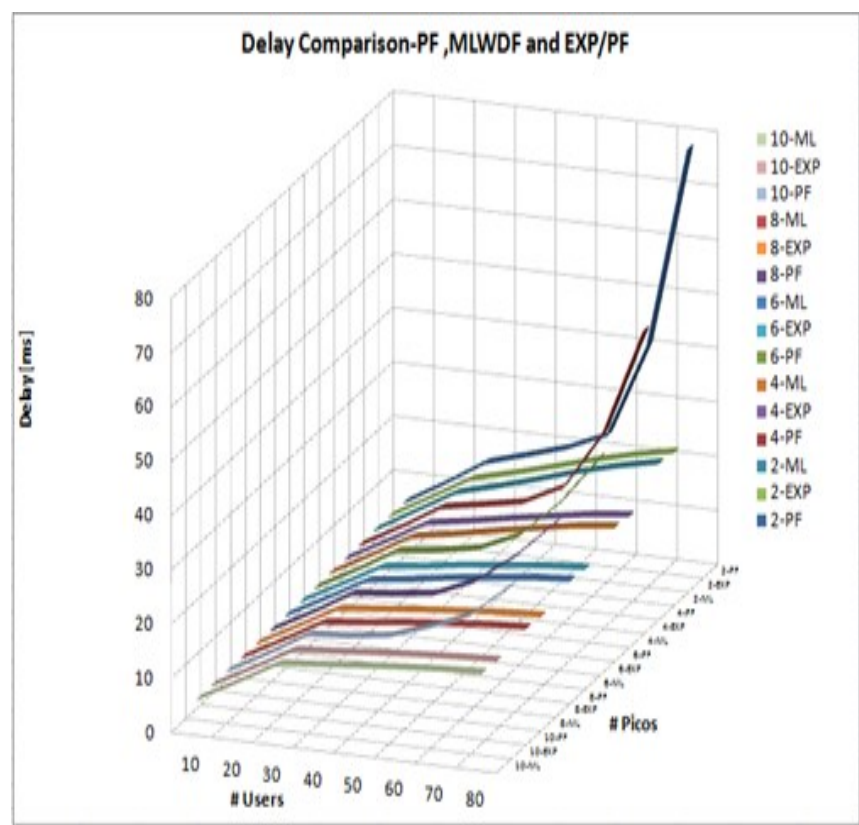

Fig.8. Delay of video traffic Comparison in macro with 2-10 Picos cells

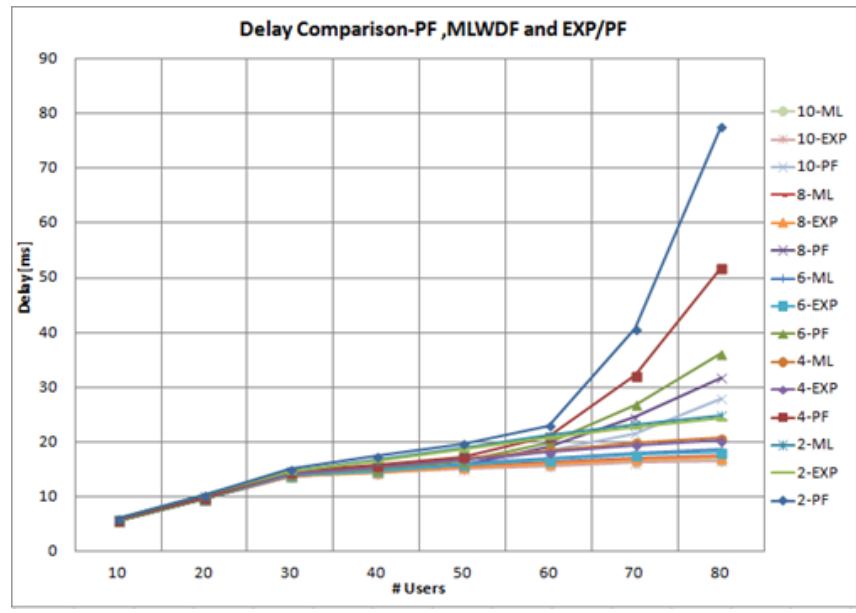

Fig.9. Comparison of delay different PS algorithms

\section{Fairness Index}

The fairness index has to be closer to the value of one. Adding more user's affects this value and graph indicates sloping down in Figure.10. The Figure 10 illustrates the 
fairness index of the system that has 2 to 10 Pico cells. The value slightly decreases from the value of one for the MLWDF and EXP/PF while the PF suffers further drop from the value of one. Adding more pico cells has no effect on the fairness as shown in Figure 10 and Figure 11. However, adding more pico cell slightly enhances the overall system fairness value. The results from the algorithms indicate worst scenario of the fairness index is at 8 Pico cells as observed from the simulation. At this point, PF shows the lowest fairness value when the number of users is 80 . MLWDF and EXP/PF also suffers further drop in the same point as aforementioned in $\mathrm{PF}$ as seen in Figure.10 and Figure.11. In all schemes, the values of fairness index are more likely to be identical at each point of pico cells although the PF has the lower fairness values among the other schemes. For example, MLWDF has similar values of fairness in 2, 4, 6, 8 and 10 Pico cells scenarios at any point of users $10,20,30,40,50,60,70$ and 80 .

\section{Fairness Comparison- PF ,MLWDF and EXP/PF}

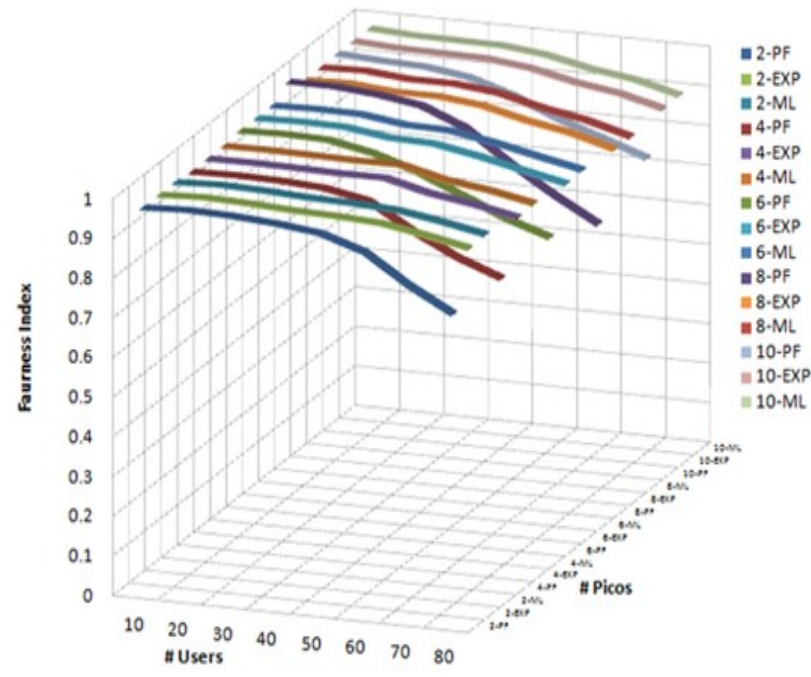

Fig.10. Fairness index in macro with 2-10 Picos cells

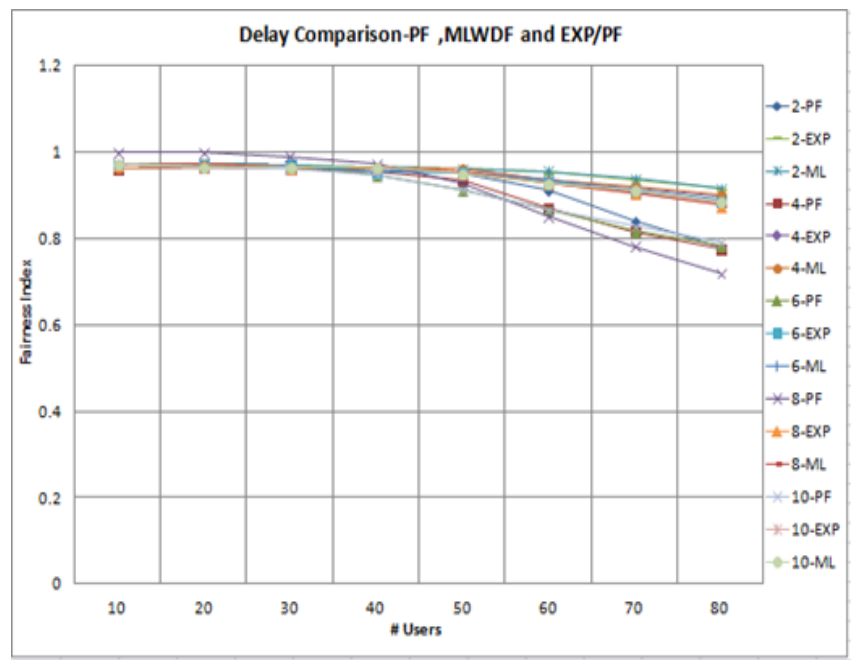

Figure 11 Fairness index in macro with 2-10 Picos cells

\section{CONCLUSION}

The PS algorithms that are developed to enhance the LTE network performance by sharing radio resources fairly among users utilizing all available resources were investigated. These algorithms depend on the traffic class and number of users. Hence, different outcomes were presented for each algorithm. To further boost the overall system performance, heterogeneous networks concept by adding small cells were used; initially with 2 Pico cells and gradually scaling up to 10 Pico cells. The gradual scaling of picos enhances throughput, PLR, decreases delay and fairness stays close to 1 . However, saturation was reached after reaching certain number of pico cells and number of users. The reason behind that is ICI limits the performance, since the same carrier frequency is used throughout the cells. The results prove that MLWDF manifests the best performance for video flows followed by EXP/PF. Further enhancement could be applied in future papers such as Almost Blank Subframes (ABS), enhanced Inter-Cell Interference Cancelation (eICIC), Cell Range Extension (CRE) concepts and using Carrier Aggregation (CA) and Coordinated Multi-Point (CoMP) within HetNets.

\section{REFERENCES}

[1] Yi. Seung June, "Radio Protocols for LTE and LTE-Advanced", Ed. New York: Wiley, September 2012, pp 20-70..

[2] B. Liu, H. Tian, and L. Xu, "An efficient downlink packet scheduling algorithm for real time traffics in LTE systems," in Consumer Communications and Networking Conference (CCNC), 2013 IEEE, 2013, pp. 364-369.

[3] Jalali, R. Padovani, and R. Pankaj, "Data throughput of CDMA-HDR a high efficiency-high data rate personal communication wireless system," in Vehicular Technology Conference Proceedings, 2000. VTC 2000Spring Tokyo. 2000 IEEE 51st, 2000, pp. 1854-1858.

[4] M. Andrews, K. Kumaran, K. Ramanan, A. Stolyar, P. Whiting, and R Vijayakumar, "Providing quality of service over a shared wireless link," Communications Magazine, IEEE, vol. 39, pp. 150-154, 2001.

[5] J.-H. Rhee, J. M. Holtzman, and D. K. Kim, "Performance analysis of the adaptive EXP/PF channel scheduler in an AMC/TDM system," Communications Letters, IEEE, vol. 8, pp. 497-499, 2004.

[6] J. Zyren and W. McCoy, "Overview of the 3GPP long term evolution physical layer," Freescale Semiconductor, Inc., white paper, 2007.

[7] Riyaj, M. R. H. Adibah, and S. Kumbesan, "Performance analysis of EXP/PF and M-LWDF in downlink 3GPP LTE system," 2009.

[8] X. Qiu and K. Chawla, "On the performance of adaptive modulation in cellular systems," Communications, IEEE Transactions on, vol. 47, pp. 884-895, 1999.

[9] H. A. M. Ramli, R. Basukala, K. Sandrasegaran, and R. Patachaianand, "Performance of well known packet scheduling algorithms in the downlink 3GPP LTE system," in Communications (MICC), 2009 IEEE 9th Malaysia International Conference on, 2009, pp. 815-820.

[10] Hu, Rose Qingyang Qian, Yi 2013, 'Comparison Femto cell and Pico cell key features”, Heterogeneous Cellular Networks (2nd Edition). 